\title{
Project SOAR's voluntary medical male circumcision portfolio
}

Project SOAR

Follow this and additional works at: https://knowledgecommons.popcouncil.org/departments_sbsr-hiv

Part of the Demography, Population, and Ecology Commons, Family, Life Course, and Society Commons, and the International Public Health Commons How does access to this work benefit you? Let us know!

\section{Recommended Citation}

Project SOAR. 2016. "Project SOAR's voluntary medical male circumcision portfolio," Activity brief. Washington, DC: Population Council. 


\section{Project's SOAR's Voluntary Medical Male Circumcision Portfolio}

Randomized controlled trials have shown that voluntary medical male circumcision (VMMC) reduces males' risk of HIV acquisition by about 60 percent, ${ }^{1}$ and a follow-on study has shown that this level of protection increases over time to reach 74 percent. $^{2}$

In March 2007 the World Health Organization (WHO) and the Joint United Nations Programme on HIV/AIDS (UNAIDS) recommended male circumcision as an additional method of HIV prevention for men, and urged countries with low male circumcision prevalence and generalized HIV epidemics to rapidly scale up VMMC programs in the context of combination HIV prevention. VMMC is now a central pillar in a multi-national strategy to reduce the transmission of HIV in high-prevalence regions of eastern and southern Africa.

Project SOAR has developed an extensive VMMC portfolio to support multiple countries with data to make informed policy and programmatic decisions about VMMC that address their specific needs and environments. The portfolio encompasses four types of activities: 1) Economic analysis of VMMC services; 2) VMMC modeling; 3) Tools and technical assistance; and 4) Dissemination.

A critical component of Project SOAR's VMMC portfolio is research utilization. At the country level, Project SOAR seeks to generate data to support planning, implementing, and monitoring VMMC programs and increase access to and use of this data through user-friendly interfaces and dissemination strategies. Simultaneously, SOAR aims to advance VMMC as a vital prevention strategy by filling important data gaps in the global discourse around VMMC.

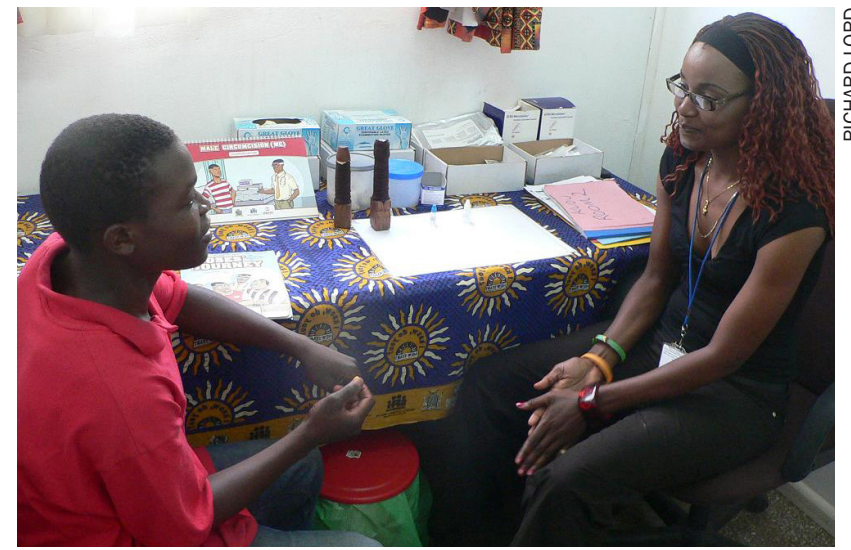

A man learning about VMMC as an HIV risk reduction strategy.

\section{Economic Analysis of VMMC Services}

\section{The Cost of VMMC Service Provision by Private Providers in South Africa}

Private practitioners have offered VMMC to clients for a number of years and are able to do so in a safe, efficient, and cost-effective way. Private sector providers present a strategic potential to increase access to VMMC services, as they attract clients who may not necessarily go to public facilities for services, thereby reaching pockets of the population that may have otherwise gone unreached.

The private sector unit cost for VMMC service provision, however, and the cost variation across the private sector are not well known. Additionally, the cost drivers are not sufficiently understood. Project SOAR is conducting a costing analysis of VMMC service provision at 10 private sector facilities in three provinces (Gauteng, Mpumalanga and KwaZulu Natal) as a follow-on to a recently completed VMMC costing study through public facilities in South Africa. This information will be used by the 
government, insurance companies, and donors for informing decisions about appropriate reimbursement for private sector providers in the future.

May 2016 - February 2017

\section{Data for Decision-Making by Private Insurance and Private Providers in Namibia and South Africa}

In some countries, the private sector represents a unique opportunity for expanding, scaling up, and sustaining VMMC services. National or private health insurance schemes often cover a substantial number of citizens, whether under schemes set up through formal employment or open schemes available to all. This work will involve reviewing how much insurance schemes reimburse the procedure costs for circumcision in the private sector, exploring areas where efficiencies can be gained, and making recommendations on a workable public-private partnership for the national VMMC program involving roles and responsibilities based on areas of relative comparative advantage.

September 2016 - February 2017

\section{VMMC Modeling}

\section{Population Estimates for VMMC Planning in Mozambique, Namibia, Kenya, and Lesotho}

Recent analyses in several sub-Saharan African countries have prompted reexamination of the target age groups and subnational regions for country VMMC strategies and operational plans. To help governments in the four countries address current implementation realities and strategically plan and monitor their programs, Project SOAR is providing impact and target population size estimates for VMMC, disaggregated by province or district and age. These estimates are based on the DMPPT 2.1 model, which has previously been used in other countries in partnership with ministries of health, PEPFAR teams, VMMC implementers, and other stakeholders. The results of the modeling exercise in these four countries will be presented to in-country stakeholders for use in VMMC planning.

December 2014 - September 2016

\section{Tools and Technical Assistance}

\section{Geographic Information System Dashboard for VMMC in South Africa and Tanzania}

Project SOAR seeks to leverage available geocoded VMMC data in South Africa and Tanzania to develop and pilot a geographic information system (GIS) dashboard. Using data visualization techniques, the VMMC GIS dashboard will allow PEPFAR teams, ministries of health, National AIDS Control Programs, and VMMC implementers to access available sub-national data on important program indicators. These indicators can include VMMC coverage, need, and costs; population density; HIV prevalence and incidence; service utilization; and health facility staffing and stock-outs in 'real time.' This will facilitate strategic planning and resource allocation decisions.

December 2015 - February 2017

\section{Online Tool for VMMC Planning}

Project SOAR is generating population size estimates and projected VMMC targets at national and district levels for about 10 of the 14 VMMC priority countries ${ }^{11}$ using the DMPPT 2.1 model, a program planning tool that disaggregates impact and population estimates by age. This requires the collection of country data to revise and update the figures and projections in countries that have already undertaken applications using a previous version of the model. SOAR also is creating a simple, webbased tool and accompanying manual that will be made publicly available for use. This online tool will allow VMMC stakeholders to look up VMMC targets, coverage, and impact projections for each country.

November 2015 - December 2017

\footnotetext{
${ }^{1}$ WHO and UNAIDS identified 13 priority countries for scale-up of VMMC: Botswana, Kenya, Lesotho, Malawi, Mozambique, Namibia, Rwanda, South Africa, Swaziland, Uganda, the United Republic of Tanzania, Zambia and Zimbabwe. PEPFAR is supporting activities to implement VMMC in these 13 countries and also in Ethiopia (Gambella province only), making a total of 14 priority countries.
} 


\section{Dissemination}

\section{Voluntary Medical Male Circumcision for HIV Prevention: New Mathematical Models for Prioritizing Sub-Populations by Age and Geography}

PLOS Collections-July/August 2016 http://collections.plos.org/vmmc2016

This PLOS Collection features new mathematical models that can help country decision-makers examine the potential effects of targeting subpopulations for VMMC services. Using these models can lead to more informed choices about where best to invest scarce resources to prevent HIV. The collection was produced in collaboration with USAID, the World Bank, and the Bill \& Melinda Gates Foundation and includes several articles written by Project SOAR principal investigators as noted below.

\section{Assessing Progress, Impact, and Next Steps in Rolling Out Voluntary Medical Male Circumcision for HIV Prevention in Fourteen Priority Countries in Eastern and Southern Africa as of 2015}

Katharine Kripke ${ }^{1}$, Emmanuel Njeuhmeli ${ }^{* 2}$, Samuelson, J. ${ }^{3}$, Melissa Schnure ${ }^{4}$, Shona Dalal ${ }^{3}$, Timothy Farley ${ }^{5}$, Catherine Hankins ${ }^{6}$, Anne G. Thomas ${ }^{7}$, Jason Reed ${ }^{8}$, Peter Stegman ${ }^{1}$, Naomi Bock. $^{9}$

${ }^{1}$ Project SOAR (Supporting Operational AIDS Research), Avenir Health, Washington, DC; ${ }^{2}$ U.S. Agency for International Development, Washington, DC; ${ }^{3}$ World Health Organization, Geneva, Switzerland; ${ }^{4}$ Project SOAR, PalIadium Group, Washington, DC; ${ }^{5}$ Sigma3 Services, Nyon, Switzerland; ${ }^{6}$ Amsterdam Institute for Global Health and Development, Amsterdam, the Netherlands; ${ }^{7}$ Naval Health Research Center, US Department of Defense, San Diego, CA; ${ }^{8}$ Jhpiego, Washington, DC; ${ }^{9}$ U.S. Centers for Disease Control and Prevention, Atlanta, GA.
The Cost of Voluntary Medical Male Circumcision in South Africa

Michel Tchuenche ${ }^{1}$, Eurica Palmer ${ }^{2}$, Vibhuti Haté $^{3}$, Ananthy Thambinayagam ${ }^{4}$, Dayanund Loykissoonlal ${ }^{5}$, Emmanuel Njeuhmeli ${ }^{6}$, Steven Forsythe $^{1}$

${ }^{1}$ Health Policy Project, Project SOAR (Supporting Operational AIDS Research), Avenir Health, Washington, DC; ${ }^{2}$ Health Policy Project, Palladium Group Consultant; ${ }^{3}$ George Washington University, Washington, DC; ${ }^{4}$ USAID, Pretoria, South Africa; ${ }^{5}$ National Department of Health, Pretoria, South Africa; ${ }^{6}$ USAID, Washington, DC.

Impact and Cost of Scaling Up Voluntary Medical Male Circumcision for HIV Prevention in the Context of the New 90-90-90 HIV Treatment Targets

Katharine Kripke ${ }^{1}$, Jason Reed ${ }^{2}$, Catherine Hankins $^{3}$, Gregory Smiley ${ }^{4}$, Catey Laube ${ }^{5}$, Emmanuel Njeuhmeli ${ }^{6}$

${ }^{1}$ Project SOAR (Supporting Operational AIDS Research), Avenir Health, Washington, DC; ${ }^{2}$ Jhpiego, Washington, DC; ${ }^{3}$ Department of Global Health and Amsterdam Institute for Global Health and Development, University of Amsterdam, the Netherlands; ${ }^{4}$ UNAIDS Regional Support Team, Eastern and Southern Africa, Johannesburg, South Africa; ${ }^{5}$ Office of the U.S. Global AIDS Coordinator, Washington, DC; ${ }^{6}$ US Agency for International Development, Washington, DC.

\section{Estimating Client Out-of-Pocket Costs for} Accessing Voluntary Medical Male Circumcision in South Africa

Michel Tchuenche ${ }^{1}$, Vibhuti Haté ${ }^{2}$, Dacia McPherson ${ }^{3}$, Eurica Palmer ${ }^{3}$, Ananthy Thambinayagam ${ }^{4}$, Dayanund Loykissoonlal ${ }^{5}$, Emmanuel Njeuhmeli ${ }^{6}$, Steven Forsythe ${ }^{1}$

${ }^{1}$ Health Policy Project, Project SOAR (Supporting Operational AIDS Research), Avenir Health, Washington, DC; ${ }^{2}$ Project SOAR (Supporting Operational AIDS Research), George Washington University, Washington, DC; ${ }^{3}$ Health Policy Project, Palladium Group Consultant; ${ }^{4}$ USAID (United States Agency for International Development), Pretoria, South Africa; ${ }^{5}$ National Department of Health, Pretoria, South Africa; ${ }^{6}$ US Agency for International Development, Washington, DC. 


\section{Webinar (2015): VMMC and Sustainability}

Maintaining HIV Prevention Benefits of Male Circumcisions by Incorporating VMMC into Routine Newborn and Adolescent Health Service Delivery

"Sustainability implications for different VMMC plans"

Rachel Sanders, Katharine Kripke

This presentation, targeted to policymakers and implementers throughout the VMMC field, explored what it will take to make increases in VMMC coverage sustainable over the long term. Countries will need to choose between programs focused on circumcising newborns, adolescents, or a combination of the two, based on a number of factors, including cost, implementation, feasibility, acceptability, health system capacity, and funding sources.

http://healthcommcapacity.org/wp-content/uploads/2015/07/Client-Age-and-Human-ResourceNeeds-for-Sustainability.pdf

\section{Webinar (September 2016): VMMC for HIV Prevention: Introducing New Mathematical Models}

This forthcoming webinar will present results from a series of papers published in July and August 2016 in PLOS Collections (see page 3).

\section{References}

1. Weiss HA, Halperin D, Bailey RC, Hayes RJ, Schmid G, Hankins C. Male circumcision for HIV prevention: from evidence to action? AIDS 2008; 22: 567-574.

2. Gray R, Kigozi G, Kong X, Ssempiija V, Makumbi F, Wattya, S, et al. The effectiveness of male circumcision for HIV prevention and effects on risk behaviors in a posttrial follow-up study. AIDS 2012; 26: 609-615.
Project SOAR is a five-year (September 2014-2019) cooperative agreement funded by the American people through the U. S. President's Emergency Plan for AIDS Relief through USAID (Agreement No. AID-OAA-A-14-00060). Project SOAR is able to accept funding from all USAID accounts.

Population Council leads the Project SOAR consortium in collaboration with Avenir Health, Elizabeth Glaser Pediatric AIDS Foundation, the Johns Hopkins University, Palladium, and The University of North Carolina at Chapel Hil.
Project SOAR/Population Council

4301 Connecticut Avenue, NW, Suite 280 Washington, DC 20008

Tel: +1202 2379400 e-mail: ProjectSOAR@popcouncil.org popcouncil.org/ProjectSOAR 